\title{
Nanoindentation-Induced Phase Transformations in Ge Studied by Electron Microscopy and Raman Spectroscopy
}

\author{
Songqing Wen,* Jae-il Jang, ${ }^{*}$ Michael J. Lance, ${ }^{+}$James Bentley, ${ }^{+}$and George M. Pharr* ${ }^{+}$ \\ *Dept of Materials Science \& Engineering, The University of Tennessee, Knoxville, TN 37996-2200 \\ ${ }^{+}$Metals \& Ceramics Division, Oak Ridge National Laboratory, PO Box 2008, Oak Ridge, TN 37831
}

The sequences of hydrostatic pressure-induced phase transformations in $\mathrm{Si}$ and $\mathrm{Ge}$ have been established in diamond anvil studies [1]. At sufficient pressure the normal diamond-cubic structure $\mathrm{Si}-\mathrm{I} / \mathrm{Ge}-\mathrm{I}$ transforms to the metallic $\beta$-tin structure; upon unloading, several metastable phases can form depending on the maximum load and/or unloading rate. In the case of Ge, the metastable phases are Ge-III(ST12) or Ge-IV(BC8). Since microstructural investigations of Si indents have revealed metastable crystalline phases, it was expected that $\mathrm{Ge}$ indents could demonstrate similar phenomena. However, there is no prior evidence of phase transformations from indentation loaddisplacement $(\mathrm{P}-\mathrm{h})$ curves or electron microscopy. In this study, nanoindentations were made on undoped $(100) \mathrm{Ge}$ at room temperature with triangular pyramidal indenters with centerline-to-face angles of $35^{\circ}$ (cube-corner) and $65^{\circ}$ (Berkovich) at loads of 10,50 and $80 \mathrm{mN}$ and a loading/unloading rate of $5 \mathrm{mN} / \mathrm{s}$. The indents were examined by SEM, micro-Raman analyses and TEM of cross-sections prepared by dual-beam FIB milling.

SEM and TEM images of indents made at $10 \mathrm{mN}$ maximum load revealed that the residual impression is deeper for the sharp $35^{\circ}$ indent (Figure 1). For both indents, the bend contour patterns indicate a high degree of residual stress with significant lattice rotation next to the transformed zone. The $35^{\circ}$ indent exhibits material plastically extruded out of the indent in a fashion similar to that reported for $\mathrm{Si}[2,3]$. Raman spectra were obtained from the $50-\mathrm{mN} 35^{\circ}$ indents with the use of a Dilor XY800 Microprobe at periods of 1, 20 and $44 \mathrm{~h}$ after the indentation test (Figure 2a). Besides broad bands around 150 and $270 \mathrm{~cm}^{-1}$ attributed to amorphous $\mathrm{Ge}$ (a-Ge), narrow peaks at 205, 230, 250 and $264 \mathrm{~cm}^{-1}$ were observed soon after the indentation but decay with elapsed time, as seen in the 20 and 44-h spectra. The peaks were tentatively assigned to Ge-IV (BC8) based on their similarity to those for Si-III (BC8) and to ones observed during diamond anvil experiments on Ge. A micro-Raman map of this $50 \mathrm{mN}$ indent created with the $205 \mathrm{~cm}^{-1} \mathrm{Ge}-\mathrm{IV}$ peak (Figure 2b) shows significant signal only within the indent, especially at the indenter edge locations. Mapping with the a-Ge peak (Figure 2c) indicates the extruded material has a high concentration of a-Ge. Frequencyshift micro-Raman maps were also made for the primary Ge-I peak at $\sim 300 \mathrm{~cm}^{-1}$ to characterize residual stress (Figure 3). The maps indicate compressive residual stress around the $65^{\circ}$ indent and, surprisingly, tensile stress around the $35^{\circ}$ indent. The extruded material is expected to be stress-free, so the apparent tensile stress may in fact indicate the presence of nanocrystalline Ge-I possibly formed by laser heating in a similar fashion to nanocrystals that are induced by the electron beam to form in cross-sectional TEM samples of $\mathrm{Si}$ indents [3]. Additional cross-sectional TEM characterization and in-situ Raman observations are planned to further understand the deformation mechanisms for indented Ge [4].

[1] V. Domnich and Y. Gogotsi, Handbook of Surfaces and Interfaces of Materials Vol.2 (Academic Press, New York, 2001).

[2] G.M. Pharr, W.C. Oliver, and D.S. Harding, J. Mater. Res. 6 (1991) 119.

[3] S. Wen, J. Bentley, J. Jang, I.M. Anderson and G.M. Pharr, Microsc. Microanal. 10 (suppl 2) (2004) 56.

[4] This research was sponsored by the NSF under grant number DMR-0203552 (SW, JJ, GMP), and at the ORNL SHaRE User Facility (MJL, JB, GMP) by the Division of Materials Sciences and Engineering, U.S. Department of Energy, under Contract DE-AC05-00OR22725 with UTBattelle, LLC. The authors also thank Dr. T. Tsui at Texas Instruments, Dallas for helping with FIB sample preparation. 


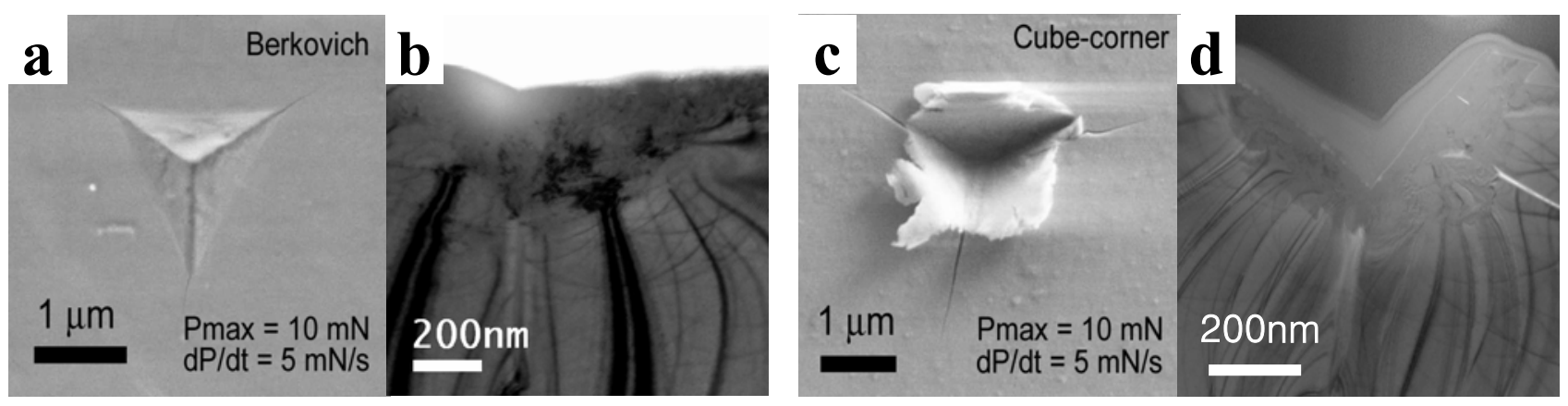

FIG. 1. (a,c) SEM and (b,d) TEM cross-sections of indents from (a,b) $65^{\circ}$ Berkovich indenter and (c,d) $35^{\circ}$ cube-corner indenter. $\left(\mathrm{P}_{\max }=10 \mathrm{mN}\right.$, Rate $\left.=5 \mathrm{mN} / \mathrm{s}\right)$
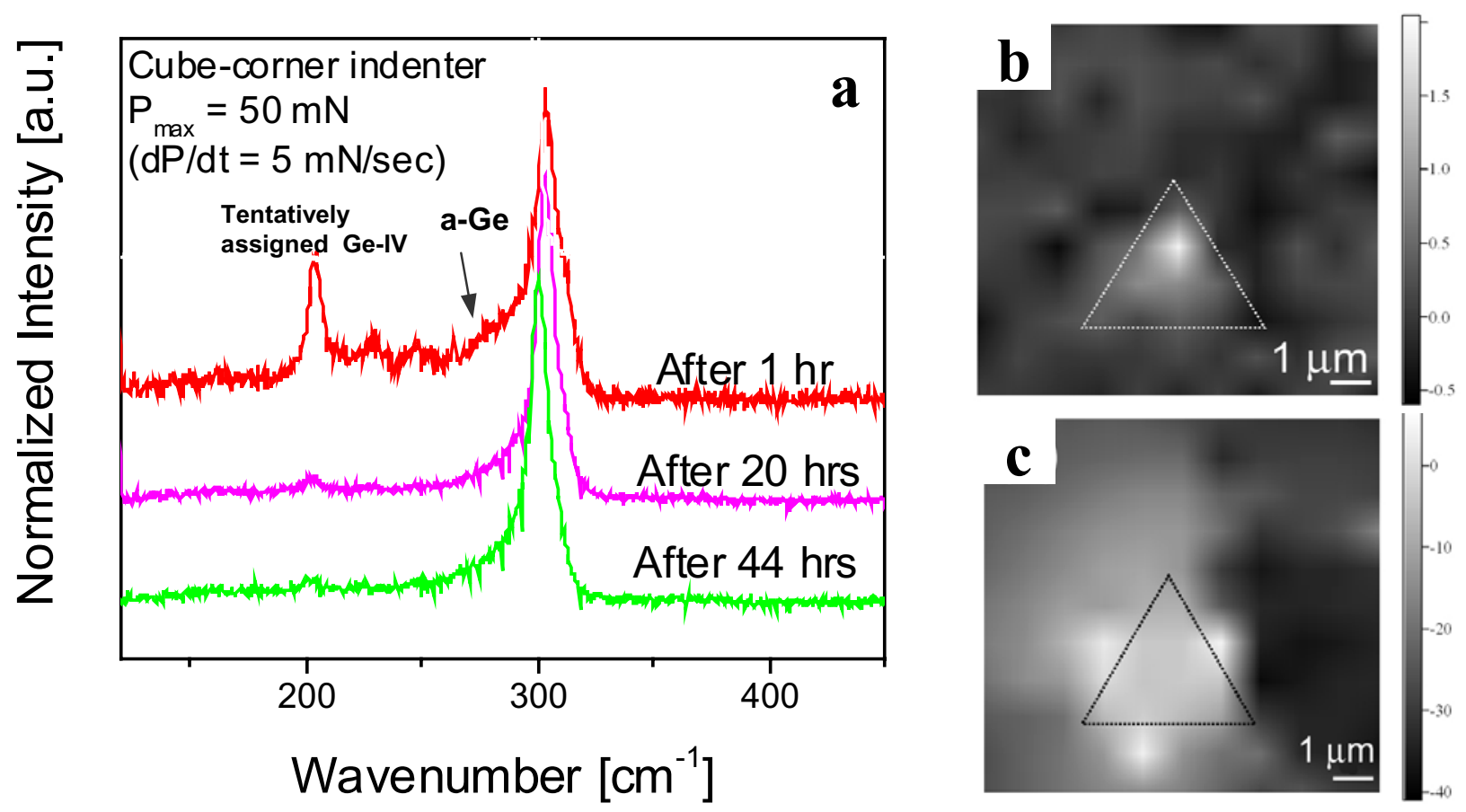

FIG. 2. Raman characterization of Ge cube-corner indent made at $5 \mathrm{mN} / \mathrm{s}$ to $P_{\max }=50 \mathrm{mN}$ (a) Raman spectra recorded 1, 20 and $44 \mathrm{~h}$ after indentation (b) Raman map at $205 \mathrm{~cm}^{-1}$ tentatively assigned to Ge-IV (c) Raman map indicating amorphous Ge.

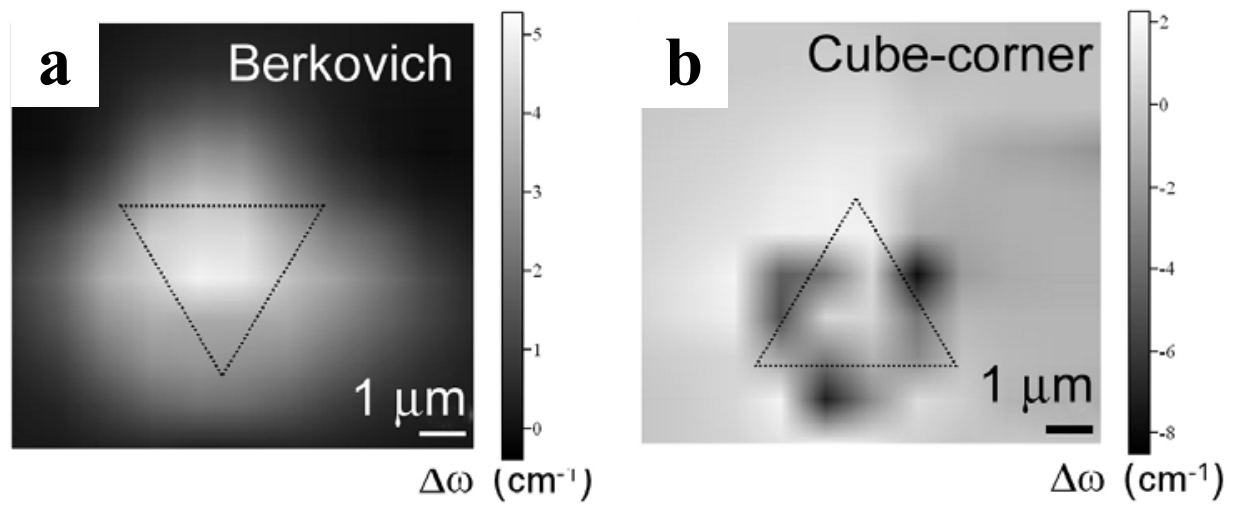

FIG. 3. Raman peak-shift maps of (a) $65^{\circ}$ Berkovich and (b) $35^{\circ}$ cube-corner indentations. $\left(\mathrm{P}_{\max }=50 \mathrm{mN}\right.$, Rate $\left.=5 \mathrm{mN} / \mathrm{s}\right)$ 